\title{
Upregulation of miR-1266-5p serves as a prognostic biomarker of hepatocellular carcinoma and facilitates tumor cell proliferation, migration and invasion
}

\author{
Yan Su${ }^{1}$, Ruizhu Xie ${ }^{1}$ and Qinyan $X^{2 \boxminus}$ \\ 'Department of Infectious Diseases, Affiliated Hospital of Weifang Medical University, Weifang 261031, Shandong, China; 2Imaging Center, Affili- \\ ated Hospital of Weifang Medical University, Weifang 261031, Shandong, China
}

\begin{abstract}
Objective: Hepatocellular carcinoma (HCC) is one of the leading causes of cancer-related death worldwide. This study aimed to analyze the prognostic value of microRNA-1266-5p (miR-1266-5p) in HCC patients and investigate its biological function in HCC progression. Methods: The expression of miR-1266-5p in tissues and cells was measured by quantitative real-time PCR (qRT-PCR). Cell counting kit-8 (CCK-8) assay was used to detect HCC cell proliferation. Transwell assay was performed to evaluate the migration and invasion of HCC cells. Kaplan-Meier methods and Cox regression analysis were used to assess the prognostic value of miR-1266-5p in HCC patients. The relationship between miR-1266-5p and DAB2IP was evaluated by luciferase reporter assay. Results: Relative expression of miR-1266-5p in tumor tissues, tissues from patients with advanced TNM stage (III-IV) and HCC cells was increased compared with that in corresponding control group. MiR-1266-5p expression was significantly associated with tumor size and TNM stage in HCC patients. Elevated expression of miR-1266$5 p$ was associated with poor prognosis of HCC patients and served as an independent prognostic factor for HCC patients. Overexpression of miR-1266-5p significantly promoted, while miR-1266-5p knockdown significantly inhibited the proliferation, migration and invasion of HCC cells. DAB2IP could directly bind to the miR-1266$5 p$. Conclusion: Our findings indicated that elevated expression of miR-1266-5p can predict the poor prognosis of HCC patients, and promotes the proliferation, migration and invasion of HCC cells. Therefore, we predict that miR-1266-5p may be a novel biomarker and therapeutic target for the treatment of HCC.
\end{abstract}

Keywords: MiR-1266-5p, hepatocellular carcinoma, prognosis, proliferation, migration, invasion

Received: 10 December, 2020; revised: 26 January, 2021; accepted: 27 April, 2021; available on-line: 19 May, 2021

⿶e-mail: xieqinyan_wfmuah@163.com

Abbreviations: AFP, alpha fetal protein; AJCC, American Joint Committee on Cancer; $\mathrm{BCa}$, bladder cancer; CCK-8, cell counting kit-8; Cl, confidence interval; DMEM, Dulbecco's Modifed Eagle's Medium; FBS, fetal bovine serum; HCC, hepatocellular carcinoma; $\mathrm{HR}$, hazard ratio; miR, microRNA; miRNAs, microRNAs; NC, negative control; OS, osteosarcoma; qRT-PCR, quantitative real-time PCR; SPSS, Statistical Product and Service Solutions; TNM, Tumor Node Metastasis

\section{INTRODUCTION}

Hepatocellular carcinoma (HCC), accounting for about $90 \%$ of primary liver cancer, is the main histo- logical subtype of liver cancer (Llovet et al., 2016). Its incidence and mortality are on the rise worldwide and it has become a major public health concern (Liang et al., 2018). The main risk factor for HCC occurrence is chronic hepatitis $\mathrm{B}$ or $\mathrm{C}$ virus infection, and other risk factors include excessive alcohol consumption, various metabolic disorders (hemochromatosis, porphyria, von Gierke disease, etc.), environmental exposure (aflatoxin), and primary biliary cirrhosis (Eatrides et al., 2017; Wallace et al., 2015). Although surgical resection is curative, early detection and therapy are still difficult in most patients with HCC, and the high recurrence rate of HCC after surgery leads to poor clinical prognosis (Teng et al., 2020; Jiang et al., 2019). Therefore, it is urgent to explore effective treatment methods and improve the prognosis of HCC patients.

MicroRNAs (miRNAs) are small noncoding RNAs that can regulate gene expression by the suppression of target mRNA translation and/or promotion of mRNA degradation (Chava et al., 2020). MiRNAs have been found to be involved in a variety of cellular processes, including cell proliferation, migration and invasion of normal and tumor cells, by regulating the expression of target genes (Deng et al., 2018; Yang et al., 2018). In addition, a large number of aberrantly expressed miRNAs might participate in the occurrence and development of tumor diseases, might be used in cancer targeted therapy, and was closely related to the prognosis of patients (Alizadeh et al., 2019; Liu et al., 2018). Thus, miRNAs play crucial roles in clinical significance and biological functions in different types of tumor diseases. Therefore, the discovery and exploration of more functional miRNAs is of great significance for the treatment of human tumor diseases, including HCC. Some abnormal miRNAs, such as miR-182-5p (Cao et al., 2018b) and miR-125a-5p (Xu et al., 2019), have been found to be closely related to tumorigenesis and prognosis in HCC. Importantly, a study on the screening of diagnostic and prognostic markers for HCC identified upregulated miR-1266-5p expression in HCC ( $\mathrm{Lu}$ et al., 2017). A recent systematic bioinformatics analysis of liver cancer biomarkers demonstrated the abnormal expression of miR-1266-5p in liver cancer, which might be associated with the prognosis of HCC (Shen et al., 2020). However, whether it can serve as an independent prognostic marker for HCC still needs further support from clinical sample data. In addition, in view of the abnormal expression level of miR-1266-5p in HCC, it is necessary to further study its biological function in the progression of HCC, so as to further understand miR-1266-5p and provide new molecular target for the prognosis and treatment of HCC. 
Therefore, this study detected the expression of miR1266-5p in HCC tissues and cells, evaluated its prognostic value in HCC patients and analyzed its biological function in the progression of HCC by cell experiment. This study might provide a new biomarker and therapeutic target for the treatment of HCC.

\section{MATERIALS AND METHODS}

\section{Patients and tissue sample collection}

A total of 132 HCC patients who underwent surgical resection at Affiliated Hospital of Weifang Medical University between 2011 and 2014 were recruited. None of the patients received any antitumor therapy prior to the surgery. Tumor tissues of patients were collected during the surgery and the corresponding normal tissues adjacent to the cancer were collected as control group, all of which were quickly stored in liquid nitrogen at $-80^{\circ} \mathrm{C}$. All the tissue samples were identified by histopathological examination and Tumor Node Metastasis (TNM) staging was conducted according to the 7 th edition of the American Joint Committee on Cancer (AJCC) cancer staging manual, including 76 cases of stage I-II and 56 cases of stage III-IV. The demographic and clinical indicators of HCC patients were shown in Table 1, including age, gender, tumor size, alpha fetal protein (AFP) levels, cirrhosis and TNM stage. All patients were followed up by telephone for 5 years, and their survival information was recorded for subsequent survival analysis. The experimental procedures of this study were approved by the Ethics Committee of Affiliated Hospital of Weifang Medical University and the included patients had signed informed consent prior to surgery.

\section{Cell culture and cell transfection}

A normal hepatocyte cell line L02 and four HCC cell lines, including Li7, Hep3B, Huh7 and SNU449, were purchased from Cell Bank of the Chinese Academy of Science (Shanghai, China). The cell lines were cultured in Dulbecco's Modified Eagle's Medium (DMEM; Gibco, NY, USA) supplemented with $10 \%$ fetal bovine serum (FBS, Gibco, NY, USA), and maintained in a $5 \% \mathrm{CO}_{2}$ atmosphere at $37^{\circ} \mathrm{C}$. Then Hep3B and Huh7 cells were transfected with miR-1266-5p mimic, mimic negative control (mimic NC), miR-1266-5p inhibitor or inhibitor NC using Lipofectamine 3000 reagent (Invitrogen) according to the manufacturer's protocols. After $48 \mathrm{~h}$ of transfection, subsequent cell experiments were performed.

\section{RNA extraction and quantitative real-time PCR (qRT-PCR)}

Total RNA was extracted from HCC tissues and cells by TRIzol reagent (Invitrogen, Carlsbad, CA, USA). The single-stranded cDNA was synthesized by reverse transcription with PrimeScript Reverse Transcriptase reagent kit (TaKaRa, Shiga, Japan).

Then the expression of miR-1266-5p was measured by qRT-PCR, which was carried out using SYBR Green PCR Master Mix (Applied Biosystems, USA) and a 7300 Real-Time PCR System (Applied Biosystems, USA). U6 was used as internal reference of miR-1266-5p, and the relative expression level of miR-1266-5p was calculated using $2^{-\Delta \Delta \mathrm{Ct}}$ method.
Table 1. Association of miR-1266-5p expression with clinical characteristics of patients with HCC

\begin{tabular}{|c|c|c|c|c|}
\hline \multirow{2}{*}{ Features } & \multirow{2}{*}{$\begin{array}{l}\text { No. } \\
n=132\end{array}$} & \multicolumn{2}{|c|}{ miR-1266-5p expression } & \multirow{2}{*}{$P$ values } \\
\hline & & Low $(n=62)$ & High $(n=70)$ & \\
\hline \multicolumn{4}{|c|}{ Age (years) } & \multirow{3}{*}{0.611} \\
\hline$\leq 50$ & 52 & 23 & 29 & \\
\hline$>50$ & 80 & 39 & 41 & \\
\hline \multicolumn{4}{|l|}{ Gender } & \multirow{3}{*}{0.768} \\
\hline Female & 55 & 25 & 30 & \\
\hline Male & 77 & 37 & 40 & \\
\hline \multicolumn{4}{|c|}{$\begin{array}{l}\text { Tumor size } \\
(\mathrm{cm})\end{array}$} & \multirow{3}{*}{0.002} \\
\hline$\leq 5$ & 71 & 42 & 29 & \\
\hline$>5$ & 61 & 20 & 41 & \\
\hline \multicolumn{4}{|l|}{$\begin{array}{l}\text { AFP } \\
(\mu \mathrm{g} / \mathrm{L})\end{array}$} & \multirow{3}{*}{0.114} \\
\hline$\leq 400$ & 67 & 36 & 31 & \\
\hline$>400$ & 65 & 26 & 39 & \\
\hline \multicolumn{4}{|l|}{ Cirrhosis } & \multirow{3}{*}{0.732} \\
\hline No & 51 & 23 & 28 & \\
\hline Yes & 81 & 39 & 42 & \\
\hline \multicolumn{4}{|c|}{ TNM stage } & \multirow{3}{*}{0.003} \\
\hline$|-| \mid$ & 76 & 44 & 32 & \\
\hline III-IV & 56 & 18 & 38 & \\
\hline
\end{tabular}

AFP, alpha fetal protein.

\section{Cell counting kit-8 (CCK-8) assay}

The proliferation ability of Hep3B and Huh7 cells was detected using CCK-8 assay. The CCK-8 reagent contains WST8, which is reduced by intracellular dehydrogenases to produce an orange yellow formazan dye that dissolves in the tissue culture medium. The amount of formazan generated is directly proportional to the number of viable cells. In this study, the stably transfected HCC cell lines were seeded in a 96-well plate at a density of $5 \times 10^{3}$ cell/well for culture, and 10 $\mu \mathrm{L}$ CCK-8 reagent was added to the cells and cultured for 24,48 and $72 \mathrm{~h}$, respectively. The absorbance at $450 \mathrm{~nm}$ was measured using a microplate reader (BioRad Laboratories, Inc.) to analyze the proliferation ability of HCC cells.

\section{Cell migration and invasion assay}

Transwell assay was performed using Transwell chambers with $8 \mathrm{~m}$ pore size membranes (Corning, USA) to investigate the effects of miR-1266-5p on cell migration and invasion. The invasion assay was carried out with the chambers coated with Matrigel, and the migration assay was carried out with the chambers without Matrigel. The bottom chamber was DMEM containing $10 \%$ FBS. The stably transfected cells $\left(5 \times 10^{3}\right.$ cell/well $)$ were seeded in the upper chamber with serum-free DMEM. After incubation at $37^{\circ} \mathrm{C}$ with $5 \% \mathrm{CO}_{2}$ for $24 \mathrm{~h}$, the cells in the bottom chamber were stained. The number of migratory or invasive cells in five randomly selected fields were counted using an inverted light microscope (Olympus Corporation) with 
A

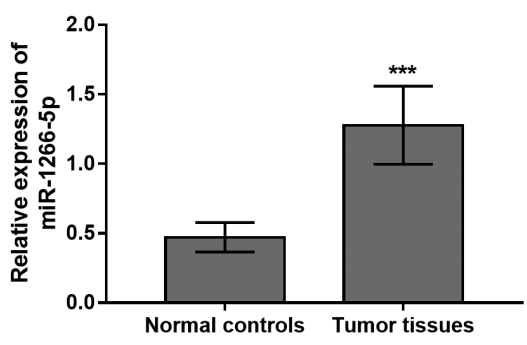

B

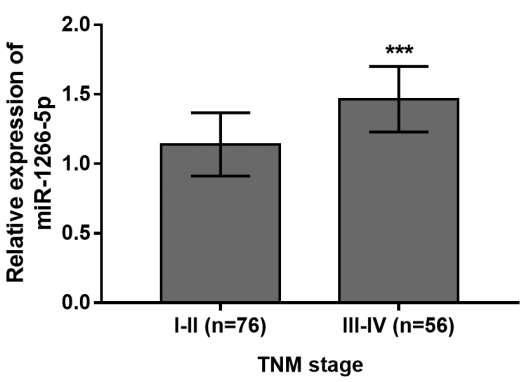

Figure 1. Expression of miR-1266-5p in tissues from patients with HCC.

A. Relative expression of miR-1266-5p in tumor tissues and normal controls; B. Relative expression of miR-1266-5p in tissues from patients with advanced TNM stage (III-IV) and early TNM stage (I-II). ${ }^{* * *} P<0.001$ vs. normal controls or tissues from patients with early TNM stage (I-I).

a magnification of $200 \times$. Each experiment was performed at least three times.

\section{Luciferase reporter assay}

To further explore the related mechanisms underlying the role of miR-1266-5p in HCC progression, the binding sequence of DAB2IP to miR-1266-5p was predicted by using TargetScan (http://www.targetscan. org/vert_72/). The 3'-UTR sequences of wild-type WT-DAB2IP and mutant MUT-DAB2IP were cloned into pmirGLO luciferase reporter vector (Promega, USA). Synthetic vectors and miR-1266-5p mimics were co-transfected into Hep3B and Huh7 cells. The relative luciferase activity was examined after $48 \mathrm{~h}$, and the results of this analysis were normalized to the luciferase activity of Renilla.

\section{Statistical analysis}

All data in this study were analyzed using Statistical Product and Service Solutions (SPSS) 21.0 software (SPSS, Inc., Chicago, USA) and GraphPad Prism 7.0 software (Inc., Chicago, USA), and were presented as mean \pm Standard Deviation (S.D.). The data of different groups were compared using Student's t-test or one-way ANOVA. The comparison between miR1266-5p expression and clinicopathological data was performed by Chi-square test. Kaplan-Meier methods and log-rank test was used for survival analysis. Cox regression analysis was used to assess the prognostic value of miR-1266-5p in HCC patients. Differences with $P<0.05$ were considered statistically significant.

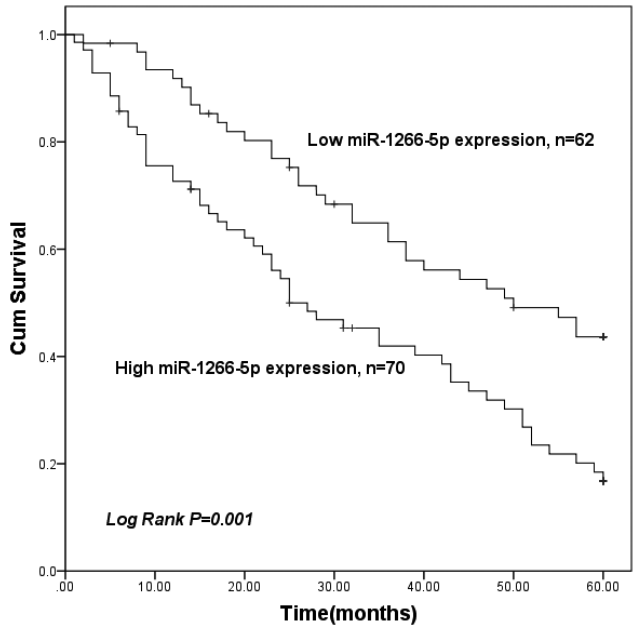

Figure 2. Kaplan-Meier survival curves for the patients with HCC.

Patients with high miR-1266-5p expression had shorter survival time than the patients with low miR-1266-5p expression (LogRank $P=0.001$ ).

\section{RESULTS}

\section{Expression of miR-1266-5p in tissues from patients with HCC}

The expression of miR-1266-5p was measured by qRT-PCR. As shown in Fig. 1A, in HCC patients' the

Table 2. Cox analysis for miR-1266-5p in HCC patients

\begin{tabular}{lllllll}
\hline \multirow{2}{*}{ Variables } & \multicolumn{2}{l}{ Univariate analysis } & \multicolumn{3}{l}{ Multivariate analysis } \\
\cline { 2 - 6 } & HR & $95 \% \mathrm{Cl}$ & P values & HR & $95 \% \mathrm{Cl}$ & $P$ values \\
\hline miR-1266-5p & 2.274 & $1.371-3.208$ & 0.004 & 2.359 & $1.462-3.807$ & $<0.001$ \\
\hline Age & 1.311 & $0.841-2.087$ & 0.221 & 1.290 & $0.836-1.990$ & 0.250 \\
\hline Gender & 1.139 & $0.731-1.709$ & 0.512 & 1.175 & $0.755-1.827$ & 0.475 \\
\hline Tumor size & 1.496 & $0.920-2.116$ & 0.087 & 1.422 & $0.915-2.210$ & 0.117 \\
\hdashline AFP & 1.199 & $0.841-1.408$ & 0.113 & 1.284 & $0.908-1.865$ & 0.108 \\
\hline Cirrhosis & 1.307 & $0.821-1.956$ & 0.263 & 1.037 & $0.667-1.613$ & 0.873 \\
\hline TNM stage & 1.931 & $1.098-3.222$ & 0.009 & 1.954 & $1.152-3.417$ & 0.019 \\
\hline
\end{tabular}

$\mathrm{HR}$, hazard ratio; $\mathrm{Cl}$, confidence interval; AFP, alpha fetal protein. 
A

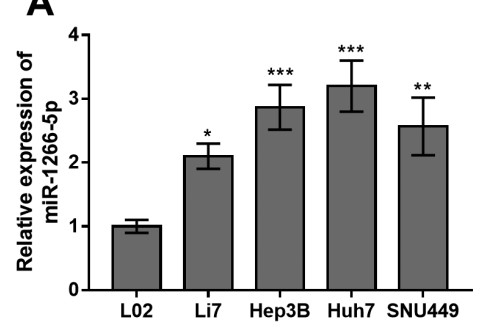

C

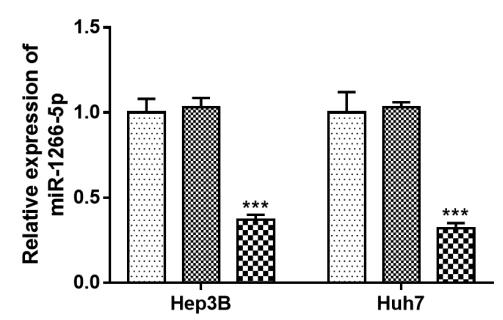

Untransfected $m$ inhibitor NC miR-1266-5p inhibitor
B

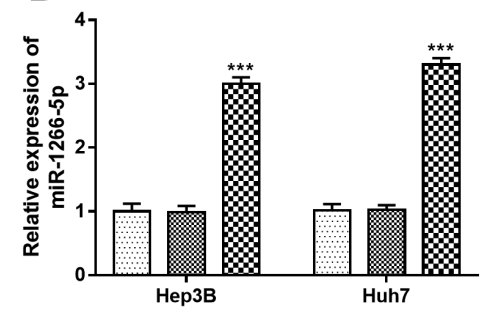

Untransfected mimic NC $\infty$ miR-1266-5p mimic

Figure 3. Relative expression of miR-1266-5p in HCC cell lines.

A. Relative expression of miR-1266-5p in normal cell line L02 and HCC cell lines Li7, Hep3B, Huh7 and SNU449. B. The miR-1266-5p expression was significantly upregulated by miR-1266-5p mimic in Hep3B and Huh7 cells. C. The miR-1266-5p expression was significantly downregulated by miR-1266-5p inhibitor in Hep3B and Huh7 cells. ${ }^{*} P<0.05,{ }^{*} P<0.01,{ }^{* *} P<0.001$ vs. L02 cell line or untransfected cells.

A
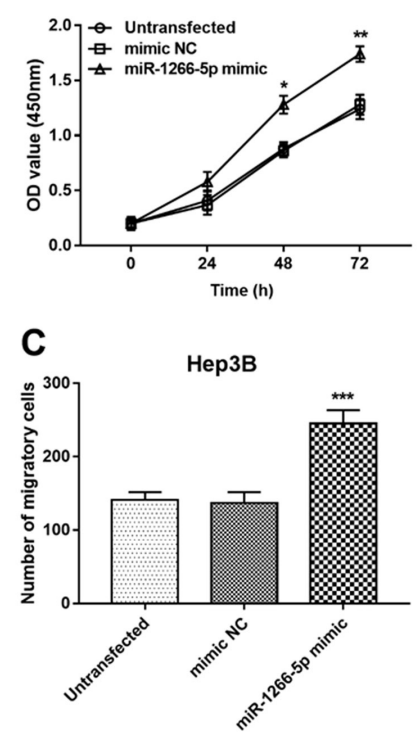

F

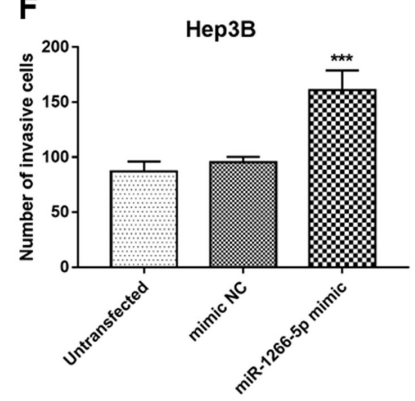

B

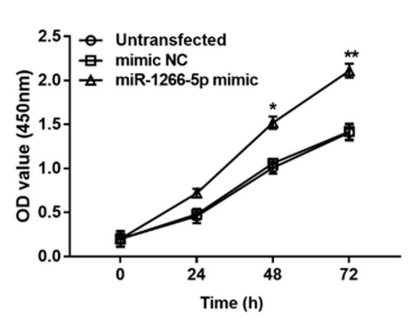

D

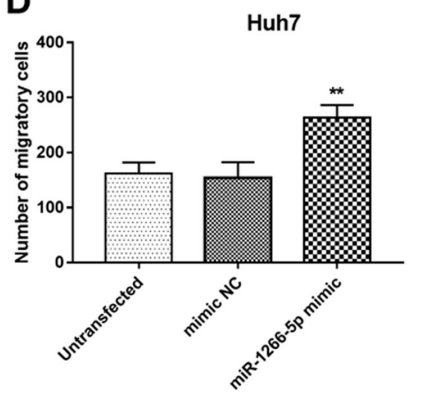

G

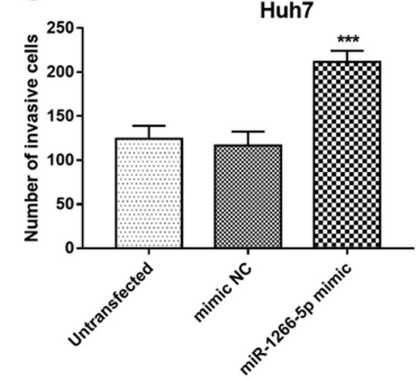

$\mathbf{E}$

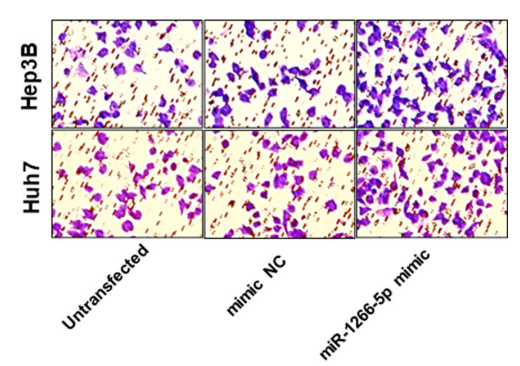

H

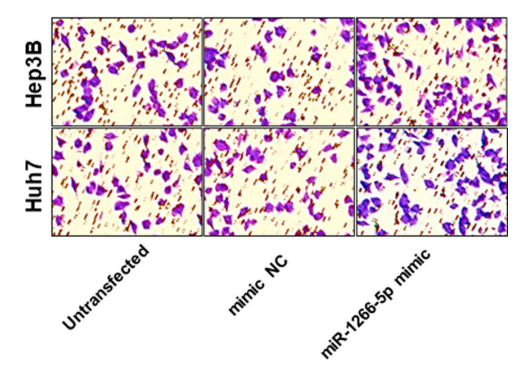

Figure 4. Overexpression of miR-1266-5p promoted HCC cell proliferation, migration and invasion.

The proliferation in both Hep3B $(\mathbf{A})$ and Huh7 (B) cells, the migration in both Hep3B and Huh7 (C-E) cells and the invasion in both Hep3B and Huh7 $(\mathbf{F}-\mathbf{H})$ cells were all significantly promoted by miR-1266-5p overexpression. ${ }^{*} P<0.05,{ }^{* *} P<0.01,{ }^{* * * P}<0.001$ vs. untransfected cells. 
relative expression of $\mathrm{miR}-1266-5 \mathrm{p}$ was upregulated in tumor tissues compared with that in corresponding normal tissues adjacent to the cancer $(P<0.001)$. Besides, the relative expression of miR-1266-5p was increased in tumor tissues from patients with advanced TNM stage (III-IV) compared with that in tumor tissues from patients with early TNM stage (I-II) (Fig. 1B, $P<0.001)$.

\section{Association between miR-1266-5p expression and clinical characteristics in patients with HCC}

The expression level of miR-1266-5p was divided into high $(n=70)$ and low $(n=62)$ expression groups using median, and the association between miR-1266$5 \mathrm{p}$ expression level and clinical characteristics of HCC patients was shown in Table 1 . No significant differences were found between miR-1266-5p expression and age, gender, AFP and cirrhosis (all P>0.05), while we found that miR-1266-5p expression was significantly associated with tumor size $(P=0.002)$ and TNM stage $(P=0.003)$ of HCC patients.

\section{High miR-1266-5p expression predicted poor survival prognosis in patients with HCC}

As shown in Fig. 2, the Kaplan-Meier survival curves indicated that patients with high miR-1266-5p expression had shorter survival time than the patients with low miR-1266-5p expression (log-rank $P=0.001$ ), sug- gesting that high miR-1266-5p expression was associated with poor survival in HCC patients. From the univariate analysis results presented in Table 2, miR-1266-5p (hazard ratio $(\mathrm{HR})=2.274,95 \%$ confidence interval $(\mathrm{CI})=1.372-3.208, P=0.004)$ and $\mathrm{TNM}$ stage $(\mathrm{HR}=1.931,95 \%$ CI $=1.098-3.222, \quad P=0.009)$ were found to be related with the overall survival of HCC patients. The multivariate COX regression analysis showed that the expression of miR-1266-5p $(\mathrm{HR}=2.359,95 \% \mathrm{CI}=1.462-3.807, P<0.001)$ and $\mathrm{TNM}$ stage $(\mathrm{HR}=1.954,95 \% \mathrm{CI}=1.152-3.417, P=0.019)$ were independently associated with survival prognosis of $\mathrm{pa}-$ tients and were independent prognostic factors for patients with HCC.

\section{Expression of miR-1266-5p in HCC cell lines}

The expression levels of miR-1266-5p were significantly increased in four HCC cell lines Li7, Hep3B, Huh7 and SNU449 compared with that in normal cell line L02 (Fig. 3A, all $P<0.05$ ). Then Hep3B and Huh7 cell lines were selected for subsequent cell experiments, due to the particularly prominent increase of miR-1266$5 \mathrm{p}$ expression level. In Hep3B and Huh7 cells, the expression level of miR-1266-5p was significantly upregulated by miR-1266-5p mimic (Fig. 3B, all $P<0.001$ ), while was significantly downregulated by miR-1266-5p inhibitor (Fig. 3C, all $P<0.001)$.
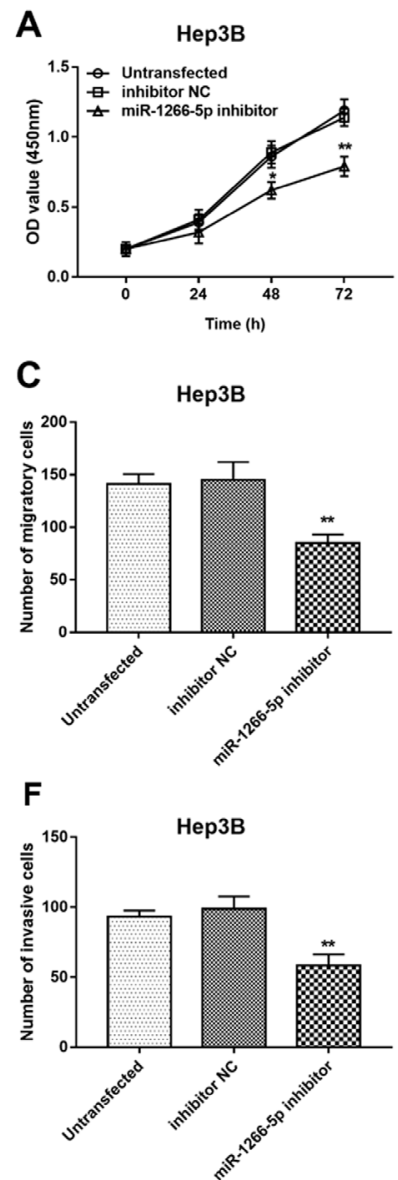

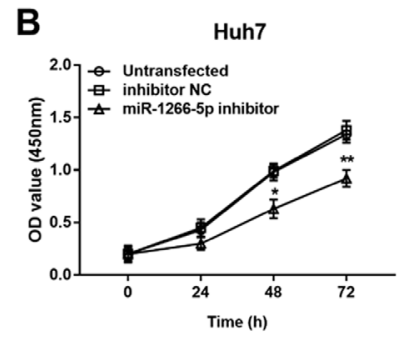

D

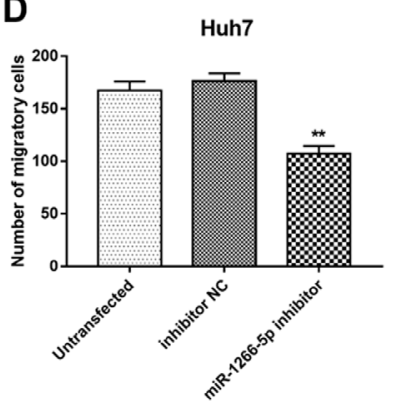

E

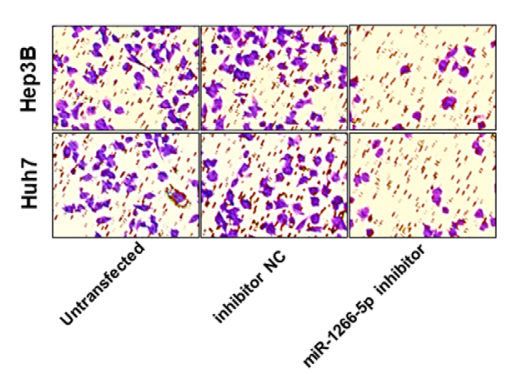

G

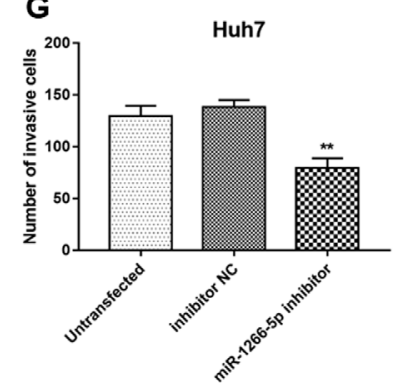

H

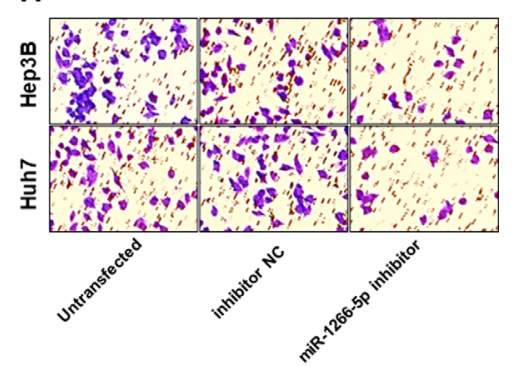

Figure 5. Knockdown of miR-1266-5p inhibited HCC cell proliferation, migration and invasion.

The proliferation of Hep3B (A) and Huh7 (B) cells, the migration of Hep3B and Huh7 (C-E) cells and the invasion of Hep3B and Huh7 (F-H) cells were all significantly inhibited by miR-1266-5p knockdown. ${ }^{*} P<0.05,{ }^{* *} P<0.01$ vs. untransfected cells. 


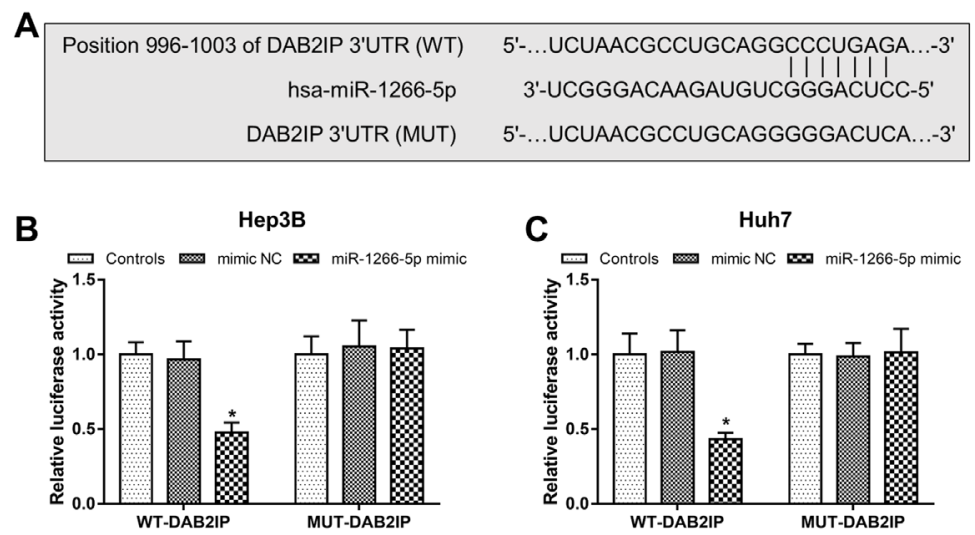

Figure 6. Direct binding of DAB2IP to miR-1266-5p.

A. The binding sequences between DAB2IP and miR-1266-5p. In Hep3B (B) and Huh7 cells (C), miR-1266-5p overexpression inhibited the relative luciferase activity in WT-DAB2IP group, whereas no changes were observed in luciferase activity in MUT-DAB2IP group. ${ }^{*} P<0.05$ vs. Controls.

\section{Overexpression of miR-1266-5p promoted HCC cell proliferation, migration and invasion}

As shown in Fig. 4A and B, miR-1266-5p overexpression significantly promoted the proliferation in both Hep3B and Huh7 cells (all $P<0.05$ ). In addition, miR1266-5p overexpression could significantly promote the migration in both Hep3B and Huh7 cells (Fig. 4C-E, all $P<0.01)$. Moreover, miR-1266-5p overexpression could significantly promote the invasion in both Hep3B and Huh7 cells (Fig. 4F-H, all $P<0.001$ ).

\section{Knockdown of miR-1266-5p inhibited HCC cell proliferation, migration and invasion}

To confirm the regulatory effects of miR-1266-5p on HCC cell biological processes, we explored the changes of proliferation, migration and invasion in both Hep3B and Huh7 cells with miR-1266-5p reduction. In both Hep3B and Huh7 cells, the knockdown of miR-1266$5 p$ expression could significantly inhibit the proliferation (Fig. $5 \mathrm{~A}$ and $\mathrm{B}$, all $P<0.05$ ), migration (Fig. 5C-E, all $P<0.01$ ) and invasion (Fig. 5F-H, all $P<0.01$ ).

\section{Direct binding of DAB2IP to miR-1266-5p}

The binding sequences between DAB2IP and miR1266-5p was shown in Fig. 6A. In Hep3B (Fig. 6B) and Huh7 cells (Fig. 6C), the relative luciferase activity in WT-DAB2IP group was inhibited by miR-1266-5p overexpression (all $P<0.05$ ), whereas no changes were observed in luciferase activity in MUT-DAB2IP group (all $P>0.05)$.

\section{DISCUSSION}

Many studies have shown that the occurrence and development of HCC is a complex process involving a large number of molecular disorders, in which miRNAs are important components (Han et al., 2018). In addition, with more and more abnormal expression patterns of miRNAs observed in tumors, the biological functions of miRNAs in tumors have received more and more attention (Dassow \& Aigner, 2013). For example, Wang and others (Wang et al., 2018) found that decreased miR-454$3 p$ and miR-374b-5p expression was associated with the low overall survival rate of bladder cancer (BCa) patients and could suppress the invasion and migration of $\mathrm{BCa}$ cells. The miR-136 expression, which was downregulated in osteosarcoma (OS) tissues and cells, played important role in the progression of OS (Chu et al., 2019). A study by Fan et al. revealed that miR-125a was downregulated in cervical cancer patients and significantly inhibited the growth, invasion and metastasis of cervical cancer cells (Fan et al., 2015). The above studies indicated that miRNA was closely related to the progression of cancers. In the past few decades, some miRNAs with abnormal expression profiles have been identified to be associated with the progression of HCC and described as functional molecules that may be associated with of HCC progression. For example, the expression of miR-182-5p which was increased in HCC tissues enhanced the proliferation and invasion of HCC cells (Cao et al., 2018a). Xu et al. showed that miR-125a-5p could inhibit the HCC progression and may be a therapeutic target for HCC patients (Xu et al., 2019). A study found that miR-200c-5p expression, decreased in HCC tissues and cells, inhibited the proliferation, migration, invasion of HCC cells and might be a therapeutic target for HCC patients ( $\mathrm{Li}$ et al., 2017). Therefore, dysregulated miRNA may play a key role in HCC progression.

It has been found that miR-1266-5p expression is altered in some other types of cancer. For instance, the expression of miR-1266-5p was downregulated in tissues and cell lines of prostate cancer (Ostadrahimi et al., 2018). However, serum miR-1266-5p levels were significantly upregulated in psoriasis patients compared to that in healthy controls (Jinnin, 2015). Thus, the expression of miR-1266-5p varied with cancer types. Importantly, studies have reported the elevated expression of miR1266-5p in HCC tissues (Lu et al., 2017) and liver cancer (Shen et al., 2020). In the present study, we measured the expression of miR-1266-5p by qRT-PCR. Similarly, we found that the miR-1266-5p expression was upregulated in tumor tissues compared with that in normal controls, was upregulated in tissues from patients with advanced TNM stage (III-IV) compared with that in tissues from patients with early TNM stage (I-II), and was upregulated in HCC cell lines compared with that in normal hepatocyte cell lines. Besides, miR-1266-5p expression was observed to be significantly associated with tumor size and TNM stage of HCC patients. Thus, we considered that miR-1266-5p might be involved in the occurrence and development of HCC. 
Considering the upregulation of miR-1266-5p in HCC tissues and cells, we further explored its prognostic value in HCC. At present, emerging studies have highlighted the high prognostic value of miRNAs in human cancers (Bertoli et al., 2015; Huang et al., 2018). Additionally, some miRNAs as prognostic biomarkers have been found in HCC, such as miR-1203 (Shi et al., 2020) and miR-503-5p (Jiang \& Li, 2019). Notably, Shen et al. revealed that miR-1266-5p might be significantly related to liver cancer prognosis (Shen et al., 2020). Thus, we plotted Kaplan-Meier survival curves, indicating the significant correlation between high miR-1266-5p expression and poor prognosis of patients with HCC. By multivariate COX regression analysis, we found that miR-1266$5 \mathrm{p}$ expression was an independent prognostic factor for HCC patients.

After studying the clinical significance of miR-1266-5p in the prognosis of HCC patients, we studied its biological functions in the progression of HCC through cell experiments. The results of our study demonstrated that overexpression of miR-1266-5p significantly promoted, while knockdown of miR-1266-5p significantly inhibited the proliferation, migration and invasion of HCC cells. Consequently, the findings revealed that miR-1266-5p might have cancer inhibitory effects on the HCC progression. In addition, the function of miR-1266-5p on the cell proliferation, migration and invasion has also been found in prostate cancer (Sun et al., 2019) and cervical cancer (Wang, Liu, et al., 2018). Notably, we further explore the target of miR-1266-5p. We found the binding sequences between DAB2IP and miR-1266-5p, and the relative luciferase activity in W'T-DAB2IP group was inhibited by miR-1266-5p overexpression. In addition, Wang and others (Wang et al., 2018) have found that miR-1266 promoted proliferation, migration and invasion of cervical cancer cells by targeting DAB2IP. Moreover, Zhang and others (Zhang et al., 2012) showed that low expression of DAB2IP was closely related to the malignant development and poor prognosis of HCC. Therefore, we speculated that miR-1266-5p may play an important role in HCC progression by targeting DAB2IP, which is an underlying molecular mechanism. However, the hypothesis of this molecular mechanism needs further study to be confirmed. Therefore, a limitation of this study is the lack of a more detailed analysis of molecular mechanisms, which is the focus of our future study. The small study cohort of this study is also a limitation, and future studies with a large study cohort are needed. In addition, cell cycle and apoptosis analyses after transfection were not performed here and will be studied in future in-depth studies on the relevant mechanisms of miR-1266-5p.

\section{CONCLUSION}

In summary, our study indicated that the expression of miR-1266-5p was increased in HCC tissues and cells, elevated expression of miR-1266-5p could predict the poor prognosis of HCC patients and significantly promoted the proliferation, migration and invasion of HCC cells. Thus, miR-1266-5p may be a novel biomarker and therapeutic target for the HCC.

\section{Acknowledgements}

Not applicable.

\section{Conflicts of Interest}

The authors have declared no conflict of interest.

\section{REFERENCES}

Alizadeh M, Safarzadeh A, Beyranvand F, Ahmadpour F, Hajiasgharzadeh K, Baghbanzadeh A, Baradaran B (2019) The potential role of miR-29 in health and cancer diagnosis, prognosis, and therapy. J Cell Physiol 234: 19280-19297. https://doi.org/10.1002/jcp.28607

Bertoli G, Cava C, Castiglioni I (2015) MicroRNAs: new biomarkers for diagnosis, prognosis, therapy prediction and therapeutic tools for breast cancer. Theranostics 5: 1122-1143. https://doi. org/10.7150/thno.11543

Cao MQ, You AB, Zhu XD, Zhang W, Zhang YY, Zhang SZ, Zhang KW, Cai H, Shi WK, Li XL, Li KS, Gao DM, Ma DN, Ye BG, Wang CH, Qin CD, Sun HC, Zhang T, Tang ZY (2018a) miR-182$5 \mathrm{p}$ promotes hepatocellular carcinoma progression by repressing FOXO3a. J Hematol Oncol 11: 12. https://doi.org/10.1186/s13045018-0555-y

Cao MQ, You AB, Zhu XD, Zhang W, Zhang YY, Zhang SZ, Zhang KW, Cai H, Shi WK, Li XL, Li KS, Gao DM, Ma DN, Ye BG, Wang CH, Qin CD, Sun HC, Zhang T, Tang ZY (2018b) Correction to: miR-182-5p promotes hepatocellular carcinoma progression by repressing FOXO3a. J Hematol Oncol 11: 56. https://doi. org/10.1186/s13045-018-0599-z

Chava S, Reynolds CP, Pathania AS, Gorantla S, Poluektova LY, Coulter DW, Gupta SC, Pandey MK, Challagundla KB (2020) miR$15 a-5 p$, miR-15b-5p, and miR-16-5p inhibit tumor progression by directly targeting MYCN in neuroblastoma. Mol Oncol 14: 180-196. https://doi.org/10.1002/1878-0261.12588

Chu Y, Hu X, Wang G, Wang Z, Wang Y (2019) Downregulation of miR-136 promotes the progression of osteosarcoma and is associated with the prognosis of patients with osteosarcoma. Oncol Lett 17: 5210-5218. https://doi.org/10.3892/ol.2019.10203

Dassow H, Aigner A (2013) MicroRNAs (miRNAs) in colorectal cancer: from aberrant expression towards therapy. Curr Pharma Design 19: 1242-1252. https://doi.org/10.2174/138161213804805739

Deng YH, Deng ZH, Hao H, Wu XL, Gao H, Tang SH, Tang H (2018) MicroRNA-23a promotes colorectal cancer cell survival by targeting PDK4. Exp Cell Res 373: 171-179. https://doi. org/10.1016/j.yexcr.2018.10.010

Eatrides J, Wang E, Kothari N, Kim R (2017) Role of systemic therapy and future directions for hepatocellular carcinoma. Cancer Control: J Moffitt Cancer Center 24: 1073274817729243. https://doi. org $/ 10.1177 / 1073274817729243$

Fan Z, Cui H, Xu X, Lin Z, Zhang X, Kang L, Han B, Meng J, Yan $Z$, Yan X, Jiao S (2015) MiR-125a suppresses tumor growth, invasion and metastasis in cervical cancer by targeting STAT3. Oncotarget 6: 25266-25280. https://doi.org/10.18632/oncotarget.4457

Han TS, Ban HS, Hur K, Cho HS (2018) The epigenetic regulation of HCC metastasis. Int J Mol Sci 19: 3978. https://doi.org/10.3390/ ijms19123978

Huang D, Peng Y, Ma K, Deng X, Tang L, Jing D, Shao Z (2018) MiR-20a, a novel promising biomarker to predict prognosis in human cancer: a meta-analysis. BMC Cancer 18: 1189. https://doi. org/10.1186/s12885-018-4907-3

Jiang SP \& Li ZR (2019) MiR-503-5p regulates cell epithelial-to-mesenchymal transition, metastasis and prognosis of hepatocellular carcinoma through inhibiting WEE1. Eur Rev Med Pharmacol Sci 23: 2028-2037. https://doi.org/10.26355/eurrev_201903_17242

Jiang Y, Han QJ, Zhang J (2019) Hepatocellular carcinoma: Mechanisms of progression and immunotherapy. World J Gastroenterol 25: 3151-3167. https://doi.org/10.3748/wjg.v25.i25.3151

Jinnin M (2015) Recent progress in studies of miRNA and skin diseases. J Dermatol 42: 551-558. https://doi.org/10.1111/13468138.12904

Li Y, Bai W, Zhang J (2017) MiR-200c-5p suppresses proliferation and metastasis of human hepatocellular carcinoma (HCC) via suppressing MAD2L1. Biomed Pharmacother 92: 1038-1044. https://doi. org/10.1016/j.biopha.2017.05.092

Liang Q, Shen X, Sun G (2018) Precision medicine: update on diagnosis and therapeutic strategies of hepatocellular carcinoma. Curr Med Chem 25: 1999-2008. https://doi.org/10.2174/0929867325666 180117101532

Liu Q, Yang W, Luo Y, Hu S, Zhu L (2018) Correlation between miR21 and miR-145 and the incidence and prognosis of colorectal cancer. Journal of B.U.ON.: official journal of the Balkan Union of Oncology 23: $29-35$

Llovet JM, Zucman-Rossi J, Pikarsky E, Sangro B, Schwartz M, Sherman M, Gores G (2016) Hepatocellular carcinoma. Nat Rev Dis Primers 2: 16018. https://doi.org/10.1038/nrdp.2016.18

Lu M, Kong X, Wang H, Huang G, Ye C, He Z (2017) A novel microRNAs expression signature for hepatocellular carcinoma diagnosis 
and prognosis. Oncotarget 8: 8775-8784. https://doi.org/10.18632/ oncotarget.14452

Ostadrahimi S, Fayaz S, Parvizhamidi M, Abedi-Valugerdi M, Hassan M, Kadivar M, Teimoori-Toolabi L, Asgari M, Shahrokh H, Abolhasani M, Mahdian R, Fard-Esfahani P (2018) Downregulation of miR-1266-5P, miR-185-5P and miR-30c-2 in prostatic cancer tissue and cell lines. Oncol Lett 15: 8157-8164. https://doi.org/10.3892/ ol.2018.8336

Shen B, Li K, Zhang Y (2020) Identification of modules and novel prognostic biomarkers in liver cancer through integrated bioinformatics analysis. FEBS Open Bio 10: 2388-2403. https://doi. org/10.1002/2211-5463.12983

Shi J, Li X, Hu Y, Zhang F, Lv X, Zhang X, Chen Q, Hu S (2020) MiR-1203 is involved in hepatocellular carcinoma metastases and indicates a poor prognosis. Neoplasma 67: 267-276. https://doi. org/10.4149/neo_2019_190414N328

Sun CM, Zhang GM. Qian HN, Cheng SJ, Wang M, Liu M, Li D (2019) MiR-1266 suppresses the growth and metastasis of prostate cancer via targeting PRMT5. Eur Rev Med Pharmacol Sci 23: 64366444. https://doi.org/10.26355/eurrev_201908_18525

Teng CF, Li TC, Huang HY, Chan WL, Wu HC, Shyu WC, Su IJ, Jeng LB (2020) Hepatitis B virus pre-S2 deletion (nucleotide 1 to 54 ) in plasma predicts recurrence of hepatocellular carcinoma after curative surgical resection. PloS One 15: e0242748. https://doi. org/10.1371/journal.pone.0242748
Wallace MC, Preen D, Jeffrey GP, Adams LA (2015) The evolving epidemiology of hepatocellular carcinoma: a global perspective. Expert Rev Gastroenterol Hepatol 9: 765-779. https://doi.org/10.1586/17474 124.2015.1028363

Wang J, Liu Y, Wang X, Li J, Wei J, Wang Y, Song W, Zhang Z (2018) MiR-1266 promotes cell proliferation, migration and invasion in cervical cancer by targeting DAB2IP. Biochimi Biophys Acta 1864: 3623-3630. https://doi.org/10.1016/j.bbadis.2018.09.028

Wang S, Zhang G, Zheng W, Xue Q, Wei D, Zheng Y, Yuan J (2018) MiR-454-3p and miR-374b-5p suppress migration and invasion of bladder cancer cells through targetting ZEB2. Biosci Rep 38: BSR20181436. https://doi.org/10.1042/BSR20181436

Xu X, Tao Y, Niu Y, Wang Z, Zhang C, Yu Y, Ma L (2019) miR125a-5p inhibits tumorigenesis in hepatocellular carcinoma. Aging 11: 7639-7662. https://doi.org/10.18632/aging.102276

Yang X, Zhang L, Song X, He W, Zhang D, Lu Q, Wu J, Wu C, Jiang J (2018) MicroRNA-613 promotes colon cancer cell proliferation, invasion and migration by targeting ATOH1. Biochem Biophys Res Commun 504: 827-833. https://doi.org/10.1016/j.bbrc.2018.09.054

Zhang X, Li N, Li X, Zhao W, Qiao Y, Liang L, Ding Y (2012) Low expression of DAB2IP contributes to malignant development and poor prognosis in hepatocellular carcinoma. I Gastroenterol Hepatol 27: 1117-1125. https://doi.org/10.1111/j.1440-1746.2011.07049.x 